\title{
GONADAL HORMONES, WOMEN AND PAIN
}

\section{Anna Maria Aloisi, Paolo Fiorenzani}

\section{Neurophysiology of Stress and Pain Laboratory, Department of Medicine, Surgery and Neurosceince, Polo Scientifico San Miniato, Siena, Italy}

\begin{abstract}
The clear presence of sex differences in chronic pain and the number of studies showing the power of gonadal hormones to modify pain-induced behavioral responses appear to have convinced clinicians and researchers. In the present review, the old and new literature is summarized to put together data on pain and its modulation by gonadal hormones, particularly estrogens. Targets of these hormones are discussed.
\end{abstract}

Key words: pain, sex differences, CNS, pain mechanisms, gonadal hormones.

Acknowledgements: the Authors thank the University of Siena.

Gonads start to produce sex hormones around the seventh week of gestation and together with the adrenals supply these products throughout life. These effects, defined as "organizational", cause permanent changes in the hormone-sensitive neural substrate to obtain a male or female subject, and they are related to both reproductive and non-reproductive functions. In addition, gonadal hormones modulate CNS functions throughout an individual's life by means of "activational" actions mostly related to their local synthesis and/or plasma concentration. Indeed the adult mammalian brain shows remarkable plasticity in response to gonadal steroid changes.

Gonadal hormone receptors are present in many structures involved in pain mechanisms. This involvement was indicated by the presence of clinical pain conditions with clear sex differences and by the changes experienced by women when hormone concentrations change in the blood [1,2,3]. For example, although prepubertal girls and boys have a similar prevalence of migraine, the lifetime prevalence of migraine becomes 3-fold higher in women than men (18\% vs 6\%) after puberty, when the androgen/estrogen ratios in the two sexes drastically change [4].

Experiments to determine the possible involvement of estrogens in pain modulation did not provide conclusive evidence. Indeed, in experimental animals and in humans, these types of studies by several different investigators yielded highly equivocal results. For instance, there are studies demonstrating estro- gens as analgesic due to the longer lasting latencies in response to acute nociceptive stimuli in ovariectomized, estradiol-treated female rodents compared to hormone-depleted controls [e.g. 5,6,7], but there are also many studies reporting no effect of estradiol on acute nociceptive responses and some reporting hyperalgesia [e.g. 8,9].

Experimental and clinical data indicate the involvement of estrogens in many chronic pain syndromes. In this case, unlike acute pain, chronic pain shows a clear sex difference in most of the syndromes considered; the incidence of pain, but also its frequency and intensity, are very often higher in females than in males, in this case the pro-nociceptive role of estrogens was repeatedly shown $[1,10]$. For instance, in humans, migraine, temporomandibular joint disorder, fibromyalgia, arthritis and interstitial cystitis are more prevalent in women than in men $[10,11,12]$. These differences commonly appear after puberty, are often related to the menstrual cycle and may disappear with menopause, clearly indicating a relationship among female gonadal hormone and painful syndromes in women. A study performed in our laboratory of transsexuals undergoing hormonal treatment reported a significant increase in chronic pain conditions in male-to-female patients undergoing estradiol/antiandrogen treatment, differently from the female-to-male patients in which there was an improvement of painful condition after treatment with testosterone [13]. Similarly in experimental animals, long-lasting pain (i.e. chronic constriction in- 
jury or arthritis), always show higher pain intensity and longer duration in females than males, differences that are abolished by ovariectomy [1].

These studies suggest that the 'female' concentration/changes of estrogens, more than acting to modulate acute pain, would affect chronic pain development. A list of possible points of action is given, from the brain to the sensory receptors.

\section{GONADAL HORMONES, ESTROGENS}

Estrogens, like other steroids, can reach their target in the cell with or without specific estrogen receptors (ER) and with slow (nuclear) or rapid (membrane) action. Two main isoforms of the receptors have been identified ( $\alpha$ and $\beta$ ) and there is much information about their modulation and activity [i.e. 14]. As for many other neurotransmitters, the effects of a given estrogen likely reflect the balance between ER $\alpha$ and $\beta$ activation in the specific cell/system; indeed it was shown that activation of ER $\alpha$ and $\beta$ can produce opposing effects, for example on anxiety [15].

Both membrane and nuclear estrogen receptors are likely important in pain modulation by estrogens [16]. However, very few pain studies have attempted to distinguish between membrane and nuclear receptor-mediated effects or between ER $\alpha$ - and ER $\beta$-mediated effects of estrogens, nor is it known which estrogenic effects on pain might be mediated by the newly described estrogen receptor, a G-protein-coupled estrogen receptor (GPR30) [17]. The activation of GPR30 requires a higher estrogen concentration and presents a sexually dimorphic distribution, as shown in the hamster hypothalamus in which its expression is much higher in females than males [18].

In general, the ER $\alpha$ and ER $\beta$ located on neurons show a similar distribution but greater density in female than male rats and some cycle-related fluctuations in females; a good example is the distribution of ER on the trigeminal brain stem complex (trigeminal subnucleus caudalis) [19]. ER $\alpha$ and $\beta$ are also located in areas of the brain involved in motor control, stress, anxiety and pain, such as the hypothalamus, amygdala, periaqueductal gray (PAG) and dorsal raphe nucleus [20]. In these brain areas, estrogenic actions can be direct on pain modulation or indirect through interactions with other neurotransmitter systems.

\section{WOMEN AND PAIN}

Further support of the role of estrogens in pain modulation comes from evidence related to cyclic estrogen fluctuations in women. The menstrual cycle is driven by the action of gonadotropins and their first input is to the production of estradiol by the ovaries.
As early as 1944, Haman [21] advanced the hypothesis of a general lowering of the sensory threshold in correspondence of menses. This was substantiated by a number of studies that found the pain threshold to be highest in the follicular phase and lowest in the luteal phase [22]. Procacci et al. [23] found that diurnal variations in pain thresholds and cyclic changes covering longer periods were present in both sexes. An hypothesis to explain pain threshold variation during the menstrual cycle stated that variations in the extracellular fluid at the receptor site or increased skin impedance may change the conductance into deeper tissues and thus alter the aversiveness of the electrical stimulation [24]. This effect can probably be explained by the finding that estrogens enhanced the dermal contents of water and collagen in a study on human subjects [25]. Interestingly Morton et al. [26] noted that the mean weight increase in women due to premenstrual water retention was $1.36 \mathrm{Kg}$.

Facchinetti et al. [27] reported a blunted increase of serum concentrations of cortisol and $\nabla$-endorphin during the premenstrual phase after intravenous administration of naloxone in women with menstrual migraine. The response was normal during the follicular phase, suggesting that there was a cyclic failure of opiate tonus in patients with menstrual migraine.

The use of different tools to test the pain threshold and its variations during the menstrual cycle has shown that while the pain threshold appears to be higher in the follicular phase for almost all natural stimuli, the opposite occurs for electrical stimuli, i.e. there is a lower threshold in the follicular phase [1].

From a clinical point of view, it is known that rapid decreases of estrogen levels occurring cyclically at menstruation appear to further increase ongoing muscle and joint pain in temporomandibular disorder and rheumatoid arthritis as well as migraine headache $[28,29]$. Indeed about $10 \%$ of female migraine sufferers have menstrual migraines, i.e. headaches that regularly occur at the beginning of menstruation [30,31]. Moreover, human studies suggest that serotonin release (estimated on the basis of prolactin concentrations after administration of d-fenfluramine) is highest during the mid-cycle, intermediate during the late luteal phase and lowest during the early follicular phase [31,32]. It was suggested that a low estrogen state, as present in the early follicular phase, may be associated with decreased release of serotonin and diminished responsiveness of 5-HT1 and 5-HT2 receptors in patients with menstrual migraines.

Sex hormones can influence the morphology and functions of many central nervous system structures [3]. Indeed sex differences in neurotransmitter levels, receptor binding and responsiveness to medications acting through these pathways have been identified [33]. For instance significant effects have been shown on transmitter systems, such as the endogenous opioid systems, dopaminergic and serotonergic activity, and 
other endogenous components. For example, it has been proposed that dysfunction of dopaminergic neurotransmission may contribute to the clinical symptoms of fibromyalgia (i.e. CWP and generalized hyperalgesia), which is much more prevalent in women than in men, and that dopamine may be an important target for the treatment of fibromyalgia [33].

In experimental animals, estrogens were shown to enhance serotoninergic neurotransmission by increasing synthesis, decreasing re-uptake and decreasing degradation of serotonin [34,35], probably through the raphe magnus, a serotonergic nucleus located in the brainstem that can modulate neurotransmission in second-order afferent neurons [36].

An important modulation exerted by estrogens is that on the opioid system. The presence of ER was demonstrated in the endorphinergic neurons in the arcuate nucleus of the hypothalamus as well as in many other CNS regions involved in pain transmission and modulation [see 37]. Interestingly Piva and colleagues [38] reported that treatment with estradiol benzoate increased the number of binding sites of dihydromorphine (a specific $\mu$-receptor ligand) in the hippocampus and thalamus. The estrogen mediated effects in the hippocampus would be related to the higher cholinergic activation (i.e. higher arousal and attention) found in females than males during experimental painful condition $[39,40]$. More recently Smith et al. [41] performed positron emission tomography studies in premenopausal women during high and low estrogen states. The high estrogen state was achieved by placement of an estradiol transdermal patch during the early follicular phase and the low estrogen condition was achieved by administration of a placebo patch at the same time. Using a model of acute pain (infusion of isotonic saline into the masseter muscle), the researchers found lower pain with higher estrogen levels probably due to the significant increase in $\mu$-receptormediated neurotransmission in the thalamus, nucleus accumbens and amygdala during the high estrogen state compared with the low estrogen state.

In the spinal cord, the interaction between pain and gonadal hormones has been studied mostly with reference to pregnancy-related events. In women as well as in rodents, antinociception occurs in response to somatic as well as visceral noxious stimuli before parturition [42]. These changes in pain perception during pregnancy were attributed to sex hormones through an opioid-mediated increase of the pain threshold $[43,44]$. Indeed the progressive increase in the circulating levels of estradiol and progesterone, as occurs during pregnancy, modulates a spinal opioid analgesic system through the estrogen-sensitive neurons present in the superficial dorsal horn lamina of the spinal cord [45].

Since the distribution of ER-containing cells in lamina II corresponds to the preferential localization in the spinal cord of preproenkephalin-expressing neurons [45], it was suggested that the effect is due to the in- volvement of estrogens in the transcriptional control of opioid synthesis and of $\delta$ - and $\beta$-opioid receptors [46]. Indeed, Amandusson et al. [47] showed that estrogen administration rapidly increases spinal cord enkephalin mRNA levels in ovariectomized rats. Moreover, other neural systems have been described as being modulated by estrogens. Tang et al., [48] reported that colonic distention increased phosphorylation of the NR1 subunit of the NMDA receptor in ovariectomized rats treated with estrogen but not in rats that did not receive estrogen. Martin et al [49] reported that neuronal responses in the trigeminal nucleus caudalis and dorsal horns of $\mathrm{Cl}$ to $\mathrm{C} 3$ were increased in response to glutamic acid during the late proestrus stage (hormonal milieu of high and declining serum concentrations of estrogen) compared with other stages of the rat estrous cycle, suggesting that high or fluctuating serum concentrations of estrogen can enhance afferent sensory input through glutamatergic mechanisms.

Estrogens have a direct effect on blood vessels, promoting vasodilatation by stimulating the synthesis of vasoactive mediators such as prostacyclin and endothelium-derived NO [50]. The vascular walls contain specific high-affinity receptors for estrogens and changes in the plasma estrogen concentrations regulate the level of ER in vascular tissue [51].

Estrogen appears to be involved in pain-related effects through its vasodilatatory action. On the other hand, in some instances estrogen appears to be involved in pain processes through potentiation of the spasmogenic response of vascular smooth muscle. This action may contribute to several pain syndromes with a recognized vascular component, e.g. migraine headache, influenced by gender and by menstrual status, and the vasoconstriction observed in Raynaud's phenomenon, a disease most common in premenopausal women [52]. The decrease in the nociceptive threshold produced by norepinephrine in estradiol-treated rats was completely antagonized by sympathectomy [52]. The basis for the qualitative differences in the vasomotor responses to estrogen among different blood vessels is unclear.

Another example of the possibility of obtaining different/opposite results when testing the same tissue with inflammatory stimuli comes from the changes obtained with different E2 concentrations. Indeed it was shown that very high estrogen concentrations tend to inhibit inflammation, whereas lower levels of estrogens may produce either no effect or a proinflammatory effect $[53,54]$. Finally the idea that estrogens are involved in inflammatory processes is supported by the finding that ER $\alpha$ and $\beta$ are present in organs of the immune system, including the thymus, spleen and bone marrow; activation of each ER by estrogens differentially contributes to granulocyte-induced inflammation, T lymphocyte proliferation and phenotypic shift, suppression of NK cell cytotoxicity, and B-lymphocyte suppression and increased differentiation [55]. 


\section{CONCLUSIONS}

Estrogens are involved in pain and in particular in chronic pain increasing women's ability to put attention and increase arousal towards the painful stimuli. This is strongly confirmed by the clinical evidence of a sex difference in most chronic painful syndromes and by the changes occurring in pain features when females pass from one reproductive period to another, i.e. menses, puberty, menopause.

Estrogens act as neurotransmitters and gene regulators, and their effects are widespread in the body. This was evident a century ago, when the first neurophysiologists started to look for sex differences. However, for unclear reasons there has been a very slow approach by researchers to the consideration of gonadal hormones, and estrogens in particular, even though good knowledge of the role of these hormones in pain mechanisms would certainly help in the understanding and treatment of pain.

\section{REFERENCES}

1. Greenspan JD, Craft RM, LeResche L, et al. Studying sex and gender differences in pain and analgesia: a consensus report. Pain 2007;132:S26-S45.

2. Fillingim $R B$, King $C D$, Ribeiro-Dasilva $M C$, et al. Sex, gender, and pain: a review of recent clinical and experimental findings. $J$ Pain 2009;10:447-85.

3. Aloisi AM, Bonifazi M. Sex hormones, central nervous system and pain. Horm Behav 2006;50:1-7.

4. Lipton RB, Stewart WF, Diamond S, et al. Prevalence and burden of migraine in the United States: data from the American migraine study II. Headache 2001;41:646-57.

5. Craft RM, Ulibarri C, Leitl MD, Sumner JE. Dose- and time dependent estradiol modulation of morphine antinociception in adult female rats. Eur J Pain 2008;12,472-9.

6. Martinez-Gomez M, Cruz Y, Salas M, et al. Assessing pain threshold in the rat: changes with estrus and time of day. Physiol Behav 1994;55:651-7.

7. Stoffel EC, Ulibarri CM, Craft RM. Gonadal steroid hormone modulation of nociception, morphine antinociception and reproductive indices in male and female rats. Pain 2003;103:285-302.

8. Walf AA, Frye CA. Anti-nociception following exposure to trimethylthiazoline, peripheral or intra-amygdala estrogen and/ or progesterone. Behav Brain Res 2003;144:77-85.

9. Ji Y, Murphy AZ, Traub RJ. Estrogen modulation of morphine analgesia of visceral pain in female rats is supraspinally and peripherally mediated. J Pain 2007;8:494-502.

10. Berkley KJ. Sex differences in pain. Behav Brain Sci 1997;20:371-80.

11. Unruh AM. Gender variations in clinical pain experience. Pain 1996;65:123-67.

12. Hargreaves R. New migraine and pain research. Headache 2007; 47:S26-43.

13. Aloisi AM, Bachiocco V, Costantino A, et al. Cross-sex hormone administration changes pain in transsexual women and men. Pain 2007;132:S60-7.

14. Balthazart J, Cornil CA, Taziaux M, et al. Rapid changes in production and behavioral action of estrogens. Neuroscience 2007;138:783-91.
15. Weiser MJ, Foradori CD, Handa RJ. Estrogen receptor beta in the brain: from form to function. Brain Res Rev 2008;57:309-20.

16. Evrard HC, Balthazart J. Rapid regulation of pain by estrogens synthesized in spinal dorsal horn neurons. J Neurosci 2004;24: 7225-9.

17. Filardo EJ, Thomas P. GPR30: a seven-transmembrane-panning estrogen receptor that triggers EGF release. Trends Endocrinol Metab 2005;16:362-7.

18. Carmeci C, Thompson DA, Ring HZ, et al. Identification of a gene (GPR30) with homology to the G-protein-coupled receptor superfamily associated with estrogen receptor expression in breast cancer. Genomics 1997;45,607-17.

19. Bereiter DA, Cioffi JL, Bereiter DF. Oestrogen receptor-immunoreactive neurons in the trigeminal sensory system of male and cycling female rats. Arch Oral Biol 2005;50:971-9.

20. Shugrue PJ, Lane MV, Mechenthaler I. Comparative distribution of estrogen receptor- $a$ and $-b$ mRNA in the rat central nervous system. J Comp Neurol 1997;388:507-25.

21. Haman JO. Pain threshold and dysmenorrhea. Am J Obstet Gynaecol 1944;47:686-91.

22. Sherman JJ, LeResche L. Does experimental pain response vary across the menstrual cycle? A methodological review. Am J Physiol Regul Integr Comp Physiol 2006;291:R245-56.

23. Procacci P, Buzzelli G, Passeri I, et al. Studies on the cutaneous pricking pain threshold in man. Circadian and circatrigintan changes. Res Clin Stud Headache 1970;3:260-76.

24. Tedford WHJ, Warren DE, Flynn WE. Alteration of shock aversion threshold during the menstrual cycle. Percept Psychophys 1977; 21:193-6.

25. Brincat M, Moniz CF, Kabalan S, et al. In skin collagen content and metacarpal index after the menopause and its prevention with sex hormone replacement. Br J Obstet Gynaecol 1987;94:126-9.

26. Morton JH, Additon $H$, Addison RG, et al. A clinical study of premestrual tension. Am J Obstet Gynecol 1953;65:1182-91.

27. Facchinetti F, Fioroni L, Martignoni E, et al. Changes of opioid modulation of the hypothalamo-pituitary-adrenal axis in patients with severe premenstrual syndrome. Psychosom Med 1994:56:418-22.

28. LeResche L, Saunders K, Von Korff MR, et al. Use of exogenous hormones and risk of temporomandibular disorder pain. Pain 1997;69:153-60.

29. Brandes JL. The influence of estrogen on migraine: a systematic review. JAMA 2006;295:1824-30.

30. Martin VT, Lipton RB. Epidemiology and biology of menstrual migraine. Headache 2008;48:S124-30.

31. Cassidy EM, Tomkins E, Sharifi $N$, et al. Differing central amine receptor sensitivity in different migraine subtypes? A neuroendocrine study using buspirone. Pain 2003;101:283-90.

32. Nappi RE, Abbiati I, Luisi S, et al. Serum allopregnanolone levels relate to FSFI score during the menstrual cycle. J Sex Marital Ther 2003;29:95-102.

33. Manson JE. Pain: sex differences and implications for treatment. Metabolism 2010;59:S16-20.

34. Pecins-Thompson M, Brown NA, Kohama SG, Bethea CL. Ovarian steroid regulation of tryptophan hydroxylase mRNA expression in rhesus macaques. J Neurosci 1996;16:7021-9.

35. Bethea CL, Lu NZ, Gundlah C, Streicher JM. Diverse actions of ovarian steroids in the serotonin neural system. Front Neuroendocrinol 2002;23:41-100.

36. Chiang CY, Sessle BJ, Hu JW. Parabrachial area and nucleus raphe magnus-induced modulation of electrically evoked trigeminal subnucleus caudalis neuronal responses to cutaneous or deep Afiber and C-fiber inputs in rats. Pain 1995;62:61-8.

37. Bodnar RJ, Kest B. Sex differences in opioid analgesia, hyperalgesia, tolerance and withdrawal: central mechanisms of action and roles of gonadal hormones. Horm Behav 2010;58,72-81. 
38. Piva F, Limonta P, Dondi D, et al. Effects of steroids on the brain opioid system. J Steroid Biochem Mol Biol 1995;53:343-8.

39. Ceccarelli I, Casamenti F, Massafra $C$, et al. Effects of novelty and pain on behavior and hippocampal extracellular ACh levels in male and female rats. Brain Res 1999;815:169-76.

40. Aloisi AM, Zimmermann M, Herdegen T. Sex-dependent effects of formalin and restraint on $c$-Fos expression in the septum and hippocampus of the rat. Neuroscience 1997;1,951-8.

41. Smith YR, Stohler CS, Nichols TE, et al. Pronociceptive and antinociceptive effects of estradiol through endogenous opioid neurotransmission in women. J Neurosci 2006;26:5777-85.

42. Cogan R, Spinnato JA. Pain and discomfort thresholds in late pregnancy. Pain 1986;27:63-8.

43. Dawson-Basoa ME, Gintzler AR. Estrogen and progesterone activate spinal kappa-opiate receptor analgesic mechanisms. Pain 1996;64:608-15.

44. Gintzler AR, Liu NJ. The maternal spinal cord: biochemical and physiological correlates of steroid-activated antinociceptive processes. Prog Brain Res 2001;133:83-97.

45. Amandusson A, Hermanson O, Blomqvist A. Estrogen receptor-like immunoreactivity in the medullary and spinal dorsal horn of the female rat. Neurosci Lett 1995;196:25-8.

46. Amandusson A, Blomqvist A. Estrogen receptors can regulate pain sensitivity. Possible explanation of certain chronic pain conditions. Lakartidningen 2001;98:1774-8.
47. Amandusson A, Hallbeck M, Hallbeck AL, et al. Estrogen-induced alterations of spinal cord enkephalin gene expression. Pain 1999; 83:243-8.

48. Tang B, Ji Y, Traub RJ. Estrogen alters spinal NMDA receptor activity via a PKA signaling pathway in a visceral pain model in the rat. Pain 2008;137:540-9.

49. Martin VT, Lee J, Behbehani MM. Sensitization of the trigeminal sensory system during different stages of the rat estrous cycle: implications for menstrual migraine. Headache 2007;47: 552-63.

50. Hayashi T, Yamada K, Esaki T, et al. Effect of estrogen on isoforms of nitric oxide synthase: possible mechanism of anti-atherosclerotic effect of estrogen. Gerontology 1997;43:24-34.

51. Farhat $M Y$, Lavigne $M C$, Ramwell $P W$. The vascular protective effects of estrogen. FASEB J 1996;10:615-24.

52. Levine JD, Taiwo YO. Beta-estradiol induced catecholamine-sensitive hyperalgesia: a contribution to pain in Raynaud's phenomenon. Brain Res 1989;487:143-7.

53. Straub RH. The complex role of estrogens in inflammation. Endocr Rev 2007;28:521-74.

54. Calabrese EJ. Estrogen and related compounds: biphasic dose responses. Crit Rev Toxicol 2001;31:503-15.

55. Carlsten H. Immune responses and bone loss: the estrogen connection. Immun Rev 2005;208:194-206. 